nitiver Konfliktfähigkeit entgegenzustehen.“ (S. 70) Hier wird wieder auf ein notwendig dialektisches Denken verwiesen.

Der zweite Teil des Buches ist fachdidaktischen Prinzipien gewidmet. Die Struktur der Kapitel mit den sieben politikdidaktischen Prinzipien ist deduktiv angelegt: Von Definitionen und Begriffsbestimmungen führt der Weg immer weiter über exemplarische Unterrichtsmaterialien hin zu methodischen Lernarrangements. Vielfach entwickelt die Autorin eigene Vorschläge zur Konkretisierung für den Unterricht. Konflikt-, Problemund Handlungsorientierung, Fallprinzip, Zukunftsorientierung und politisch-moralische Urteilsbildung zählen zu den zentralen politikdidaktischen Merkmalen und kennzeichnen einen anspruchsvollen Politikunterricht.

In Teil III entwickelt Reinhardt eine Stufendidaktik für die gymnasiale Oberstufe, die unter der Zielsetzung der Wissenschaftspropädeutik steht. Die vorgeschlagenen Stufen des Lernens können als Beitrag zur aktuellen fachdidaktischen Diskussion um Kompetenzmodelle gelesen werden. Die Anbindung an Theorien der Sozialwissenschaften wird deutlich, verbunden mit Vorschlägen zu eigenen begrenzten Forschungsprojekten der Schüler. Die Methodenkompetenz wird entfaltet (empirische Studien, Experiment, Ganzschrift usw.). Letztlich sind diese Vorschläge nicht nur „Stoff“, sondern werden selbstreflexiv genutzt (zum Beispiel das Kohlberg-Modell).

Das Buch erfüllt den (hohen) Anspruch als „Praxishandbuch“ auf dreifache Weise: zum einen durch vielfältige Nutzung empirischer Studien, zum zweiten durch Rückbezüge auf eigene Praxiserfahrungen in Schule und Hochschule, zum dritten durch das Kleinarbeiten der politikdidaktischen Ziele und Prinzipien bis hin zu Materialien, Schrittfolgen und variablen Lernwegen.

Mit der Typologie der Fehlkonzepte hat Sibylle Reinhardt einen anschlussfähigen Impuls für die aktuelle Diskussion geliefert, der weiter erforscht und in der Unterrichtsplanung berücksichtigt werden sollte. Das gilt auch für das beim politischen Lernen notwendige und mehrfach angesprochene „dialektische Denken“. Das Buch bietet also zugleich eine fundierte, praxisbezogene Einführung in politikdidaktisches Denken, verweist aber darüber hinaus auf „unerledigte Hausaufgaben“ der Politikdidaktik im Kontext der Diskussion um Bildungsstandards, domainspezifische Kompetenzen und Kerncurricula der politischen Bildung.

Hans-Werner Kuhn

\title{
Autoritatives Standardwerk zur empirischen Wahlforschung
}

Falter, Jürgen W. und Harald Schoen (Hrsg.): Handbuch Wahlforschung, VS Verlag für Sozialwissenschaften, Wiesbaden 2005, 826 Seiten, € 49,90.

Um es gleich vorweg zu sagen: Dieses Buch ist ein großer Wurf. Wer sich künftig über die wesentlichen Paradigmen, Theorien, Forschungsthemen, Methoden und gesicherten, aber auch kontroversen empirischen Kenntnisse der Wahlforschung in Deutschland und 
auch international (im Sinne der in englischer Sprache zugänglichen Beiträge) informieren möchte, kommt an diesem Werk nicht vorbei. Mit eindrucksvoller Akribie und offenkundigem Anspruch, eine lückenlose Gesamtdarstellung zu liefern, wird hier eine detaillierte Bilanz einer politikwissenschaftlichen Teildisziplin geliefert, die jedem Interessenten die Möglichkeit bietet, sich rasch einen gründlichen Überblick über ihre klassischen wie aktuellen Forschungsgebiete zu verschaffen. Alles wird umfassend und bis in die kleinsten Verästelungen, gleichzeitig übersichtlich und wohl strukturiert zusammengefasst. Wie enzyklopädisch der Forschungsstand aufgearbeitet wurde, verdeutlicht das Literaturverzeichnis: Es umfasst allein 140 der über 800 eng bedruckten Seiten des Buches. Ein Glossar sowie ein Sach- und ein Autorenregister tragen zu seinem hohen Nutzwert weiter bei.

Das Werk ist in fünf Teile gegliedert. Der einleitende Block holt weit aus - über den Rahmen der empirischen Wahlforschung im engeren Sinne hinaus - und beginnt mit einer demokratietheoretischen Erörterung der Bedeutung von Wahlen als Kerninstitution der repräsentativen Demokratie. Sie definiert zunächst ihren Gegenstand - die demokratische Wahl mit ihren besonderen Eigenheiten, die sie von (Schein-) Wahlen in autokratischen Systemen abhebt - und diskutiert dann die Positionen unterschiedlicher demokratietheoretischer Perspektiven zum Institut der Wahl, wobei auch kritische Stimmen nicht ausgespart bleiben. Es folgt eine historische Abhandlung zur Geschichte des Wahlrechts und seiner Wahrnehmung durch die Bürger sowie der Entwicklung der Wahlsysteme. Zwei weitere Grundlagenkapitel diskutieren die typischen Methoden der empirischen Wahlforschung, ihre Leistungen, aber auch Probleme. Block II des Buches erörtert die zentralen theoretischen Ansätze der Wahlforschung. Nach einer Diskussion der klassischen Wahlgeographie werden die drei heute als zentral akzeptierten Schulen der Wahlforschung breit dargestellt: der auf Paul Lazarsfeld sowie Seymour Martin Lipset und Stein Rokkan zurückgehende „soziologische“ Ansatz, der auf die Michigan-Schule zurückgehende "sozialpsychologische“ sowie der von Anthony Downs begründete „ökonomische“ Ansatz. Entlang dieser drei Ansätze sind auch die Darstellungen in den restlichen Kapiteln des Buches organisiert.

Die Teile III und IV sind „speziellen Fragestellungen“ beziehungsweise „ausgewählten Gebieten" der Wahlforschung gewidmet - eine Unterscheidung, die nicht so recht einleuchtet. Einige der hier behandelten Gegenstandsbereiche sind evident. Themen wie Wahlbeteiligung beziehungsweise Nichtwahl, die Bedeutung von Wertorientierungen für das Wählerverhalten, die Rolle der Massenmedien für Wahlentscheidungen oder die Analyse von Wahlsystemen und ihren Wirkungen sind im Rahmen einer solchen Gesamtschau unverzichtbar. Andere Kapitel können als weniger selbstverständlich gelten; sie reflektieren eher die besonderen Forschungsinteressen der Autoren, die allesamt aktuell oder in der Vergangenheit an der Universität Mainz tätig sind beziehungsweise waren, etwa die Auseinandersetzung mit den Phänomenen wechselhafter Wahlentscheidungen und der Wahl extremistischer Parteien, der Bedeutung der Persönlichkeit für das Wählerverhalten und der historischen Wahlforschung. Besonders zu begrüßen ist die Aufnahme eines Kapitels über Wahlkampfforschung. Lange Zeit von der Wahlforschung fast vollständig vernachlässigt, finden die Wahlkampagnen der Parteien erst in jüngster Zeit die Aufmerksamkeit, die sie verdienen. 
Der Band schließt mit einer von Oscar W. Gabriel und Silke J. Keil verfassten Bilanz zum Stand der empirischen Wahlforschung in Deutschland, die neben der akademischen auch die kommerzielle Wahlforschung behandelt. Zu Recht wird der deutschen Wahlforschung ein hoher Leistungsstand bescheinigt. Viele kritische Kommentare früherer Jahre sind mittlerweile obsolet; einige haben sich auch aufgrund ihrer offenkundigen Zeitbedingtheit im Sinne einer Verhaftung in mittlerweile überwundenen Paradigmenzwisten von selbst erledigt. Vor diesem Hintergrund können die tatsächlichen Blindstellen der deutschen Wahlforschung heute mit kühlerem Kopf herausgearbeitet werden. Uneingeschränkt ist der Diagnose zuzustimmen, dass insbesondere die theoretischen Impulse aus der Psychologie, die in den USA im letzten Jahrzehnt nachhaltigen Erkenntnisfortschritt gebracht haben ${ }^{1}$, hierzulande noch völlig unzureichend aufgegriffen worden sind. Das hat vermutlich auch damit zu tun, dass die Ausbildung deutscher Politikwissenschaftler in der Regel keinerlei psychologische Kompetenzen vermittelt, wobei nicht nur an Theorien und Befunde des Faches zu denken ist, sondern auch an seine spezifischen Methoden, allen voran das Experiment. Zu Recht moniert wird überdies, dass die Einbindung der Wähler in soziale und politische Kontexte immer noch zu wenig beachtet wird und dass sich die Forschung zu stark auf Bundestagswahlen konzentriert und darüber die so genannten Nebenwahlen (Europa-, Landtags- und Kommunalwahlen) vernachlässigt. Dass komparative Analysen im Sinne genuiner Mehrebenenmodelle immer noch Mangelware sind, trifft ebenfalls zu, gilt aber auch für die Wahlforschung andernorts.

Durch den enzyklopädischen Charakter des vorliegenden Werkes tritt deutlich zutage, dass die deutsche Wahlforschung hoch professionell ist und einen beachtlichen Kenntnisstand erreicht hat, auch wenn sie bislang noch relativ wenig zur internationalen Theorieentwicklung beigetragen hat; hier ist sie nachhaltig von der US-Forschung geprägt. Das ist eine Folge der Sozialisationskette, die viele ihrer zeitgenössischen Vertreter mit den Gründervätern der Wahlforschung in den USA verbindet, aber auch der relativ geringen Größe der Teildisziplin in Deutschland, gerade im Vergleich zu den USA. So gibt es in Deutschland - den überzeugenden Leistungen dieser Subdisziplin zum Trotz nach wie vor keinen einzigen Lehrstuhl für Wahl- und Einstellungsforschung.

Das „Handbuch Wahlforschung“ fasst in autoritativer Weise den aktuellen Forschungsstand zusammen und wird bald als unersetzliches Standardwerk anerkannt sein. Als einführendes Lehrbuch ist es weniger zu empfehlen, da es dafür zu umfassend im Anspruch und dadurch zu komplex in der Darstellung ist. Bei einer etwaigen Neuauflage könnte aber zumindest in Erwägung gezogen werden, die Methodenkapitel - dem gelungenen Vorbild des vorzüglichen Textbuches von Joclyn Evans ${ }^{2}$ folgend - durch ein kleines Tutorial zu erweitern, das auch Nichtspezialisten in die Lage versetzen könnte, typische Ergebnispräsentationen wahlsoziologischer Forschung im Sinne einer „methods literacy“ zumindest lesen und einordnen zu können. Auch ein kommentierter Überblick über die Informations- und Datenquellen sowie Datenbestände der deutschen und der internationalen Wahlforschung, die heute über das Internet leicht zugänglich sind, könnte den Nutzwert des Buches noch weiter steigern.

Rüdiger Schmitt-Beck

1 Vgl. zuletzt Richard R. Lau / David P. Redlawsk, How Voters Decide. Information Processing during Election Campaigns, Cambridge 2006.

2 Joclyn Evans, Voters and Voting: An Introduction, London 2004. 